\title{
CORNER TRANSFER MATRICES AND QUANTUM AFFINE ALGEBRAS
}

\author{
OMAR FODA† and TETSUJI MIWA* \\ $\dagger$ Institute for Theoretical Physics, University of Nijmegen, \\ 6525 ED NIJMEGEN, The Netherlands. \\ * Research Institute for Mathematical Sciences, \\ Kyoto University, Kyoto 606, Japan
}

\begin{abstract}
Let $\mathcal{H}$ be the corner-transfer-matrix Hamiltonian for the six-vertex model in the antiferroelectric regime. It acts on the infinite tensor product $W=V \otimes V \otimes V \otimes \cdots$, where $V$ is the 2-dimensional irreducible representation of the quantum affine Lie algebra $U_{q}(\widehat{\mathfrak{s l}}(2))$. We observe that $\mathcal{H}$ is the derivation of $U_{q}(\widehat{\mathfrak{s l}}(2))$, and conjecture that the eigenvectors of $\mathcal{H}$ form the level-1 vacuum representation of $U_{q}(\widehat{\mathfrak{s l}}(2))$. We report on checks in support of our conjecture.
\end{abstract}

\section{Introduction}

\subsection{Motivation and results}

Baxter's corner transfer matrix (CTM) [B1] has been providing an effective method for computing 1-point functions in exactly-solvable lattice models: the eight-vertex model [B1], the hard hexagon model and its generalizations [B1], [ABF], [DJKMO1], and the six-vertex model and its generalizations [DJKMO2], [(KMN $)^{2}$.

These computations revealed a deep connection between lattice models and quantum affine Lie algebras, where the temperture variable in the former is the deformation parameter $q$ in the latter. Let us recall two examples of this connection.

The multiplicities of the CTM eigenvalues of the six-vertex model and its generalizations are equal to the weight multiplicities of irreducible highest weight representations of a certain affine Lie algebra [DJKMO2].

The CTM eigenvectors, in the zero temperaturte limit, coincide, as has been shown for a large class of vertex models, with the base vectors (the crystal base $[\mathrm{K}]$ ) of irreducible highest weight representations of the quantum affine Lie algebras $U_{q}(\mathfrak{g})$ at $q=0,[\mathrm{MM}],[\mathrm{JMMO}],\left[(\mathrm{KMN})^{2}\right]$.

In this paper, we search for an algebraic structure in the CTM eigenvectors at $q \neq 0$. We work in the context of a simple off-critical model: the six-vertex model in the anti-ferroelectric regime (cf. 8.10, [B1]). For this model the corresponding algebra is $U_{q}(\widehat{\mathfrak{s l}}(2))$ and the corresponding representation is the level-1 vacuum representation.

For clarity, we present our results basically in the order that we obtained them. We first observed that, though the 'bare' CTM Hamiltonian, in the large lattice 
limit, has divergent eigenvalues, one can define a 'renormalized' Hamiltonian, order by order in a low-temperature expansion, that has finite eigenvalues bounded from below. We report on that in $\S 2$.

Because of the identification, mentioned above, of the eigenvectors at $q=0$ and the crystal base, it is natural to suspect that, even at $q \neq 0$, the eigenvectors of $\mathcal{H}$ are identified with the weight vectors in the level-1 vacuum representation of $U_{q}(\widehat{\mathfrak{s l}}(2))$.

Our preliminary checks confirmed that the ground state (the lowest eigenvector of $\mathcal{H})$ satisfies the highest weight conditions of $U_{q}(\widehat{\mathfrak{s l}}(2))$, as a vector in the semiinfinite tensor product of the two-dimensional representation of $U_{q}(\widehat{\mathfrak{s l}}(2))$, on which $\mathcal{H}$ acts naturally. So we proceeded to compute the highest weight vector and the next few descendants in this tensor representation. This computation is independent of that used to compute the eigenvectors of $\mathcal{H}$. We report on that in $\S 3$.

Once we had these two, a priori independent sets of vectors, we proceeded systematically to compare them. This is discussed in $\S 4$. Our computations confirm that the eigenvectors of $\mathcal{H}$ form indeed the level-1 representation of $U_{q}(\widehat{\mathfrak{s l}}(2))$. Though non-trivial, and quite extensive, these computations are limited to a finite number of terms in a perturbation expansion, and we can only make the above statement as a conjecture.

For further support of our conjecture, we observe that $\mathcal{H}$ satisfies simple commutation relations with the generators of the quantum affine Lie algebra $U_{q}(\widehat{\mathfrak{s l}}(2))$. More precisely: $\mathcal{H}$ acts as a derivation of $U_{q}(\widehat{\mathfrak{s l}}(2))$.

In the following subsection we wish to recall the $q=0$ result.

\subsection{The low temperature limit $q=0$}

The six-vertex model, that we are interested in, is related to the quantum affine Lie algebra $U_{q}(\widehat{\mathfrak{s l}}(2))$ of Drinfeld, and Jimbo, [D] [J], since its Boltzmann weights form the $R$-matrix of the 2-dimensional representation $V$, of $U_{q}(\widehat{\mathfrak{s l}}(2))$. As we mentioned above, the deformation parameter $q$ has the physical interpretation of temperature. We restrict our consideration to the anti-ferroelectric regime: $0<$ $x<1,-1<q<0$, i.e., in particular, $\Delta=\left(q+q^{-1}\right) / 2<-1$. This is an off-critical, ordered regime. In the limit $q \rightarrow 0_{-}, \Delta \rightarrow-\infty$, it is totally ordered, and things become simpler.

Kashiwara's theory of the crystal bases of quantum groups [K]: the representation theory of $U_{q}(\widehat{\mathfrak{s l}}(2))$ at $q=0$, enables us to sum the $q$-series obtained by the CTM method, and to compute the 1-point functions $\left[(\mathrm{KMN})^{2}\right]$. We wish to elaborate this point.

Let $A(x, q)$ be a CTM for the six-vertex model. The parameter $x$ is the multiplicative spectral parameter. In the large lattice limit, the CTM has the asymptotic form $[\mathrm{B} 1]$

$$
A(x, q)=\alpha(x, q) x^{\mathcal{H}(q)} .
$$

Here $\alpha(x, q)$ is a normalization constant and $\mathcal{H}=\mathcal{H}(q)$ is the CTM Hamiltonian. 
It acts naturally on the infinite tensor product

$$
W=V \otimes V \otimes V \otimes \cdots .
$$

From the periodicity requirement for the eigenvalues of the CTM one can deduce that the eigenvalues of $\mathcal{H}$ are $0,1,2, \cdots$. The multiplicities of these eigenvalues are equal to the weight multiplicities of the level-1 vacuum representation $V\left(\Lambda_{0}\right)$ [DJKMO2]. Since the multipicity is independent of $q$, we can compute it in the limit of $q=0$. In fact, at $q=0$, the CTM Hamiltonian $\mathcal{H}$ is diagonal with respect to pure tensors in $W$.

A pure tensor, or more appropriately, a path, as we will refer to it from now on, is a vector $|p\rangle$ in $W$ of the form

$$
|p\rangle=p(1) \otimes p(2) \otimes p(3) \otimes \cdots,
$$

where $p(k)=( \pm)$ is the weight vector $(+)$ or $(-)$ in the $k$-th component $V=$ $\mathbf{Q}(q)(+) \oplus \mathbf{Q}(q)(-)$ of $W$. Think of $W$ as a semi-infinite 'spin-chain': there are two possible, equivalent anti-ferroelectric ground states. We choose to work with the one that satisfies the following boundary conditions

$$
\begin{aligned}
p(k) & =(+) \quad \text { if } k \text { is even and } \gg 0 . \\
& =(-) \quad \text { if } k \text { is odd and } \gg 0 .
\end{aligned}
$$

Let us denote by $\mathcal{P}$ the set of all paths that satisfy (1.2.4). These paths are the eigenvectors of the CTM in the low-temperature limit $q \rightarrow 0_{-}$.

In $[\mathrm{K}]$ the crystal base $B(M)$ was introduced for an integrable representation $M$ of $U_{q}(\mathfrak{g})$. It is a base of $M$ at $q=0$ that enjoys remarkably simple combinatorial properties.

Consider the level-1 vacuum representation $M=V\left(\Lambda_{0}\right)$ for $U_{q}(\widehat{\mathfrak{s l}}(2))$. There is a natural isomorphism between the set $\mathcal{P}$ and the crystal $B\left(V\left(\Lambda_{0}\right)\right)[\mathrm{MM}]$ (see also [JMMO] and $\left.\left[(\mathrm{KMN})^{2}\right]\right)$. In other words, the paths, which are the eigenvectors of the CTM at $q=0$, are at the same time the base of $V\left(\Lambda_{0}\right) \subset W$ at $q=0$.

In this work, we take a first step to extend the above result to $q \neq 0$. We infer, on the basis of direct computations, that the subspace of $W$ spanned by the eigenvectors

$$
|v\rangle=\sum_{p} c(p, v)|p\rangle
$$

of the CTM is isomorphic to $V\left(\Lambda_{0}\right)$ under the action of $U_{q}(\widehat{\mathfrak{s l}}(2))$ - the action via the infinite comultiplication on $W$.

\subsection{Related work}

In [B2], Baxter discussed the eight-vertex model. In particular, at the point where it reduces to two decoupled Ising models, the diagonalization of the CTM by 
means of the spinor representation is given. There are more results in [Da1], [TP], [IT] for the Ising case, also on the basis of the free fermion structure of the Ising model.

As for the six-vertex model, in [Da2], the diagonalization of the CTM Hamiltonian is studied in the ferroelectric phase.

Interesting observations are given in [Te]: the CTM Hamiltonian is a boost operator for the row-to-row transfer matrix (cf. $[\mathrm{SW}],[\mathrm{T}]$ ), and in $[\mathrm{SB}]$ : there is a similarity in the spectrum of the CTM in the thermodynamic limit at an off critical temperature and that of the row-to-row transfer matrix on a finite size lattice at the critical temperature. The latter was further discussed in $[\mathrm{DP}],[\mathrm{F}]$.

\section{Acknowledgements}

The first author wishes to thank the organizers of 'RIMS91 project on INFINITE ANALYSIS' for their friendly hospitality, and for the pleasant atmosphere at RIMS. We both wish to thank the participants of the project for discussions, and Brian Davies for useful communications. We are indebted to Atsushi Nakayashiki for careful reading of the manuscript.

\section{The eigenvectors of $\mathcal{H}$}

\subsection{The model}

We consider the six-vertex model in the anti-ferroelectric regime, which is one of the simpler off-critical lattice models with an exact solution [B1]. The variables of the model are arrows that live on the bonds of a lattice, and can point in either direction. In this paper we use 'signs' $( \pm)$ instead of 'arrows' as we shall explain below.

Draw the lattice diagonally, i.e., with bonds to be directed SW-NE or SE-NW. Let $(+)$ (resp. $(-))$ stand for an arrow that points upwards (resp. downwards). Figure 2.1 shows an equivalent pair of signs and arrows around a vertex. To each configuration of signs around a vertex we associate a Boltzmann weight. One can think of the signs in the upper row as an initial configuration that develops to a final configuration given by the signs in the lower row, with a probability given by the corresponding Boltzmann weight.

Figure 2.1.1 A configuration around a vertex. 
The non-vanishing Boltzmann weights, written as rotated by $45^{\circ}$ clockwise, are

$$
\begin{aligned}
& (+) \underset{(+)}{\stackrel{(+)}{\mid}}(+)=(-) \underset{(-)}{\mid}(-)=\frac{x-q^{2}}{1-q^{2}}, \\
& (-) \underset{(+)}{\mid}(-)=(+) \underset{(-)}{\mid}(+)=\frac{q(x-1)}{1-q^{2}} \text {, } \\
& (+) \underset{(-)}{\stackrel{(+)}{\mid}}(-)=x,(-) \underset{(+)}{\mid}(+)=1 .
\end{aligned}
$$

All other possible configuration have vanishing Boltzmann weights.

The parametrization (2.1.1) has the following meaning in connection with the $R$-matrix of $U_{q}(\widehat{\mathfrak{s l}}(2))$. Let $V$ be the 2-dimensional $\mathbf{Q}(q)$ vector space with the distinguished base vectors $(+)$ and $(-)$. Introduce a matrix $R(x, q)$ by

$$
R(x, q)(i) \otimes(j)=\sum_{k, l}(k) \underset{(l)}{(i)}(j)(k) \otimes(l) .
$$

Then it satisfies the Yang-Baxter equation:

$$
\begin{aligned}
& (1 \otimes R(x, q))(R(x y, q) \otimes 1)(1 \otimes R(y, q)) \\
& =(R(y, q) \otimes 1)(1 \otimes R(x y, q))(R(x, q) \otimes 1) .
\end{aligned}
$$

\subsection{Definitions}

Let $A(x, q)$ be the CTM of the SE quadrant [B1]. In the large lattice limit, it is of the form (1.2.1), where $\mathcal{H}=\mathcal{H}(q)$ acts on $W$ given by (1.2.2) with $V=$ $\mathbf{Q}(q)(+) \oplus \mathbf{Q}(q)(-)$.

Let us compute $\mathcal{H}$. Set $x=e^{\lambda}$. Note that the $R$-matrix given by (2.1.1-2) is proportional to the identity matrix at $\lambda=0$. Let $R(x, q)=1+r(q) \lambda+O\left(\lambda^{2}\right)$ be the expansion of $R(x, q)$ at $\lambda=0$. We have

$$
\mathcal{H}=\sum_{k=1}^{\infty} k r(q)_{k, k+1} .
$$

Here $r(q)_{k, k+1}$ means $r(q)$ acting on the $k$-th and the $(k+1)$-th components of $W$. We expect that the difference of any two eigenvalues of $\mathcal{H}$ is an integer.

Let us compute $\mathcal{H}$ explicitly. We denote by $\left(s s^{\prime}\right)$ the $k$-th and the $(k+1)$-th components of a vector in $W$. Define two operators $P_{k}^{\prime}$ and $Q_{k}$ that act on $\left(s s^{\prime}\right)$. The prime in $P_{k}^{\prime}$ is because this operator will be re-defined shortly. 
The diagonal operator $P_{k}^{\prime}$ is given by

$$
P_{k}^{\prime}\left(s s^{\prime}\right)=H\left(s, s^{\prime}\right)\left(s s^{\prime}\right)
$$

where

$$
\begin{aligned}
H\left(s, s^{\prime}\right)=1 & & \text { if }\left(s s^{\prime}\right)=(++),(+-),(--), \\
=0 & & \text { if }\left(s s^{\prime}\right)=(-+) .
\end{aligned}
$$

The operator $Q_{k}$ is given by

$$
Q_{k}( \pm \mp)=(\mp \pm), \quad Q_{k}( \pm \pm)=0 .
$$

In other words, it permutes adjacent spins that are different.

We also define an operator $S_{k}$ that measures spin. It acts on the $k$-th component of $W$ as

$$
S_{k}(+)=\frac{1}{2}(+), S_{k}(-)=-\frac{1}{2}(-) .
$$

From the Boltzmann weights, and the above definitions, one can show that the Hamiltonian $\mathcal{H}$ is given by

$$
\mathcal{H}=\frac{1}{1-q^{2}}\left\{\sum_{k=1}^{\infty} k\left\{P_{k}^{\prime}+q^{2}\left(P_{k}^{\prime}-1\right)\right\}+q \sum_{k=1}^{\infty} k Q_{k}-q^{2} \sum_{k=1}^{\infty} S_{k}\right\} .
$$

We call the path

$$
|\varnothing\rangle=(-+-+\cdots)
$$

the ground-state path: or the zero-temperature ground state. The point is that, as we will see, at $q \neq 0$, the true ground state (i.e., the lowest eigenvector of $\mathcal{H}$ ) consists of an infinite linear superposition of paths, with $q$-dependent coefficients. At $q=0$ the ground state reduces to the above path $|\varnothing\rangle$. The coefficients of all others vanish.

We fix the value of the spin operator $S=\sum_{k=1}^{\infty} S_{k}$ on $|\varnothing\rangle$ as $S|\varnothing\rangle=0$. Consequently, $S$ is well-defined on all the paths in $\mathcal{P}$. We call the eigenvalue of $S$ at $p$ the spin of $p$ and denote it by $s(p)$. We define

$$
\iota(p)=\min \left\{n \mid \exists i_{1}, \ldots, i_{n} \text { such that }|p\rangle=Q_{i_{1}} \cdots Q_{i_{n}}|\varnothing\rangle\right\},
$$

and

$$
l(p)=\max \{n \mid p(n) \neq \varnothing(n)\} .
$$

We refer to them as the depth and the length of $p$, respectively. The depth is the 'inversion number' of $p$ relative to the ground state path: that is, starting from the ground state path, it counts the minimal number of inversions one has to perform on neighbouring pairs $(+,-)$ or $(-,+)$ to obtain a certain path. 
The length gives the extension of spin fluctuations in $p$, as compared to the ground state path: that is, it gives the left-most spin position following which a given path coincides with the ground state path.

\subsection{The renormalized Hamiltonian}

Starting to compute the eigenvectors of the CTM Hamiltonian given above, one finds immediately that its eigenvalues are infinite. That is why we refer to $\mathcal{H}$ as the 'bare' Hamiltonian. We need to renormalize it.

First, we look for the lowest eigenvector $|\phi\rangle$. In all generality, we expect it to be of the form

$$
|\phi\rangle=\sum_{p} c(p, \phi)|p\rangle,
$$

where the sum is restricted to such paths that $s(p)=0$.

We are going to subtract an infinite scalar from $\mathcal{H}$ in such a way that the lowest eigenvalue is shifted to 0 . We will surmise that this infinite scalar can be defined consistently, order-by-order in a pertubation expansion in $q$, and refer to the final result as the 'renormalized' Hamiltonian.

The first step is to redefine $P_{k}^{\prime}$ so that the energy of the ground state path is 0 . We define $P_{k}$ by

$$
\begin{aligned}
P_{k}\left(s s^{\prime}\right) & =\left(H\left(s, s^{\prime}\right)-H(-,+)\right)\left(s s^{\prime}\right) \quad \text { if } k \text { is odd, } \\
& =\left(H\left(s, s^{\prime}\right)-H(+,-)\right)\left(s s^{\prime}\right) \quad \text { if } k \text { is even. }
\end{aligned}
$$

We define the 'classical' energy $\omega(p)$ of a path $p$ to be the eigenvalue of $P=$ $\sum_{k=1}^{\infty} k P_{k}$ at $p$. This is the actual energy of a path at $q=0$.

We suppose the renormalized Hamiltonian is of the form

$$
\mathcal{H}_{r e}=\frac{1+q^{2}}{1-q^{2}}(P+\varepsilon Q-\varepsilon q S-\varepsilon R),
$$

where

$$
Q=\sum_{k=1}^{\infty} k Q_{k}, R=\sum_{k=1}^{\infty} k R_{k}, \quad \varepsilon=\frac{1}{2 \Delta}
$$

Here $R_{k}$ is a scalar to be determined in the recursive process of computing $c(p, \phi)$. We wish to choose $R$ so that the lowest eigenvalue of $\mathcal{H}_{r e}$ is zero.

\subsection{Spin-0 paths}

Denoting the paths in terms of + and - spins is not convenient for long paths. Let us introduce a more concise notation. Given a path $p$ of spin- 0 , we define a list of non-negative integers

$$
a=[a(1), a(2), \cdots]
$$


as follows. Let us introduce the auxiliary parameters $k, s$ and $c$. We start from $k=0, a=[], s=-1, c=0$. We increase $k$. At the $k$-th process we do the following;

$$
\begin{aligned}
& \text { if } s=p(k) \text { then } \\
& \text { if } c=0 \text { then } \\
& \text { (i) add } 0 \text { to } a \text { at the last column } \\
& \text { (ii) change } s \text { to }-s \\
& \text { else } \\
& \text { (i) change } c \text { to } c-1 \\
& \text { else } \\
& \text { (i) add } c+1 \text { to } a \text { at the last column } \\
& \text { (ii) change } c \text { to } c+1
\end{aligned}
$$

The list $a$ satisfies

$$
a(i) \geq 0, a(i+1) \leq a(i)+1, a(i)=0 \text { if } i \gg 0 .
$$

The correspondence between the spin- 0 paths and the lists satisfying (2.4.2) is oneto-one. We label $p$ as $(a(i))_{1 \leq i \leq m}$ where $m=\max \{i \mid a(i) \neq 0\}$. The following are the paths of spin 0 and length less than 6.

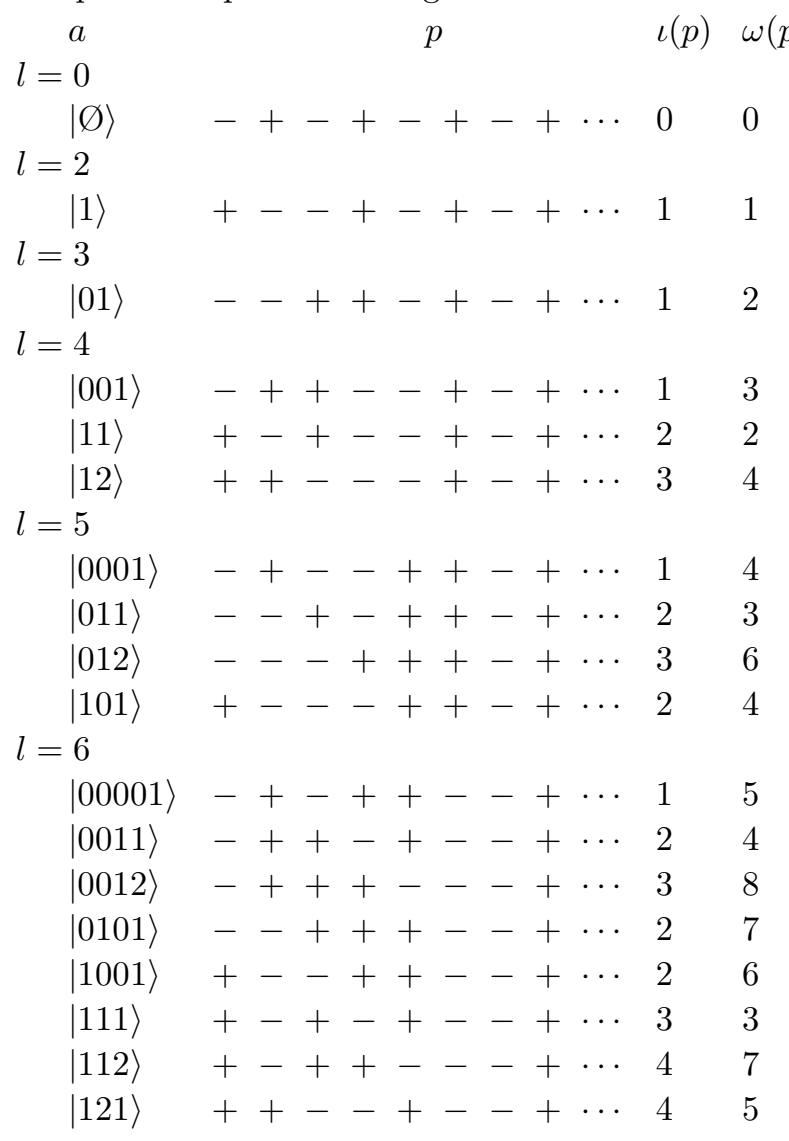




$$
\begin{array}{llll}
|122\rangle & ++-+---+\cdots & 5 & 6 \\
|123\rangle & +++-\ldots--+\cdots & 6 & 9
\end{array}
$$

\subsection{A perturbative expansion}

We are going to solve

$$
\mathcal{H}_{r e}|\phi\rangle=0
$$

perturbatively at $\varepsilon=0$. $\left(\mathcal{H}_{r e}\right.$ is given in (2.3.2).) We assume the following functional form of the coefficients in (2.3.1):

$$
\begin{aligned}
& c(\varnothing, \phi)=1, \\
& c(p, \phi)=0 \text { if } s(p) \neq 0, \\
& c(p, \phi)=\sum_{l \geq 0} c_{l}(p, \phi) \varepsilon^{2 l+\iota(p)} .
\end{aligned}
$$

We set $c_{l}(0, \phi)=0$ for later convenience. We assume also that

$$
R_{k}=\sum_{j=0}^{\infty} r_{j k} \varepsilon^{2 j+1}
$$

where the coefficients $r_{j k}$ are yet unknown.

We define the operators $Q_{k}^{+}$and $Q_{k}^{-}$as follows.

$$
\begin{aligned}
Q_{k}^{ \pm}|p\rangle & =Q_{k}|p\rangle \text { if } Q_{k}|p\rangle \neq 0 \text { and } \iota\left(Q_{k} p\right)=\iota(p) \pm 1 \\
& =0 \quad \text { otherwise. }
\end{aligned}
$$

Example. If $|p\rangle=|011\rangle$, the expression $c_{0}\left(Q_{k}^{+} p, \phi\right)+c_{1}\left(Q_{k}^{-} p, \phi\right)$ means 0 , $c_{1}(0001, \phi), c_{0}(012, \phi), c_{1}(01, \phi), 0, \ldots$ when $k=1,2,3,4,5, \ldots$, respectively.

With this notations, (2.5.1) reads as

$$
\begin{aligned}
\omega(p) c_{l}(p, \phi) & +\sum_{k=1}^{\infty} k\left\{c_{l-1}\left(Q_{k}^{+} p, \phi\right)+c_{l}\left(Q_{k}^{-} p, \phi\right)\right\} \\
& -\sum_{k=1}^{\infty} \sum_{j=0}^{\infty} k r_{j k} c_{l-1-j}(p, \phi)=0 .
\end{aligned}
$$

Let us determine the unknown terms $r_{j k}$. Consider (2.5.3) for $|p\rangle=|\varnothing\rangle$. Then, by using (2.5.2) we have

$$
\sum_{k} k c_{l-1}(\underbrace{0 \cdots 0}_{k-1} 1, \phi)=\sum_{k} k r_{l-1 k}
$$


Therefore, we choose

$$
R_{k}=c(\underbrace{0 \cdots 0}_{k-1} 1, \phi)
$$

or equivalently

$$
r_{j k}=c_{j}(\underbrace{0 \cdots 0}_{k-1} 1, \phi) .
$$

With this choice the recursion equation (2.5.3) becomes self-contained. It can be solved self-consistently if and only if the succesive coefficients satisfy the asymptotic factorizability: for any $j$ the equality

$$
c\left(Q_{k}^{+} p, \phi\right) \equiv c(\underbrace{0 \cdots 0}_{k-1} 1, \phi) c(p, \phi) \bmod \varepsilon^{j}
$$

holds for sufficiently large $k$. In fact, if (2.5.5) is valid (and that is certainly the case according to our extensive computer checks), we can truncate the summation on $k$ in (2.5.3) at a sufficiently large value of $k$, and compute $c_{l}(p, \phi)$ independently of the cut-off.

Example. Let us illustrate how we perturbatively solve (2.5.3). Take $|p\rangle=|1\rangle$. Then, for $l=0,(2.5 .3)$ reads as $c_{0}(1, \phi)+c_{0}(\varnothing, \phi)=0$. Therefore, we have $c_{0}(1, \phi)=-1$. Similarly, we have $r_{0 k}=-1$ for all $k$. Therefore, for $l=1,(2.5 .3)$ reads as

$$
\begin{aligned}
c_{1}(1, \phi) & +c_{1}(\varnothing, \phi)+3 c_{0}(11, \phi)+4 c_{0}(101, \phi)+5 c_{0}(1001, \phi)+\cdots \\
& +(1+2+3+4+5+\cdots) c_{0}(1, \phi)=0 .
\end{aligned}
$$

Using that $c_{1}(\varnothing, \phi)=0, c_{0}(1, \phi)=-1, c_{0}(11, \phi)=2, c_{0}(101, \phi)=1, c_{0}(1001, \phi)=$ $1, \ldots$, which are computable prior to $c_{1}(1, \phi)$, we have $c_{1}(1, \phi)=0$.

\subsection{Excited states}

Take $s \in \mathbf{Z}$ and $\omega \in \mathbf{Z}_{>0}$. An excited state with spin $s$ and energy $\omega$ is a formal $\operatorname{sum}|v\rangle=\sum_{p} c(p)|p\rangle$ satisfying

$$
\begin{aligned}
& S|v\rangle=s|v\rangle, \\
& \mathcal{H}_{r e}|v\rangle=\omega|v\rangle .
\end{aligned}
$$

Let us denote the space of such vectors by $H_{s, \omega}$.

Suppose that there are $m$ distinct paths $p_{1}, p_{2}, \ldots, p_{m}$ that have the same values of the spin and the classical energy. Then, one can expect that there are $m$ distinct solutions to the eigenvalue equations (2.6.1-2). We use the following Ansatz: choose $i \in\{1,2, \ldots, m\}$, and impose that

$$
\begin{aligned}
& c\left(p_{j}\right)=\delta_{i j}, \\
& c(p)=\sum_{l \geq 0} c_{l}(p) \varepsilon^{\iota\left(p, p_{i}\right)+2 l} .
\end{aligned}
$$


Here we denote by $\iota\left(p, p^{\prime}\right)$ the inversion number of $p$ relative to $p^{\prime}$. For example, $\iota(-+++-+\cdots,++-+-+\cdots)=2$.

The recursion equation reads as

$$
\begin{aligned}
(\omega(p) & -\omega) c_{l}(p)+\sum_{k}\left(c_{l-1}\left(Q_{k}^{+} p\right)+c_{l}\left(Q_{k}^{-} p\right)\right) \\
& -\sum_{k} \sum_{j=0}^{l-1} k r_{j k} c_{l-1-j}(p)=\sum_{j=1}^{l}\left(\omega a_{j}+s b_{j-1}\right) c_{l-j}(p) .
\end{aligned}
$$

Here

$$
\sqrt{1-4 \varepsilon^{2}}=\sum_{j=0}^{\infty} a_{j} \varepsilon^{2 j}\left(=\frac{1-q^{2}}{1+q^{2}}\right), \frac{1-\sqrt{1-4 \varepsilon^{2}}}{2 \varepsilon}=\sum_{j=0}^{\infty} b_{j} \varepsilon^{1+2 j}(=q) .
$$

We denote the eigenvector obtained from (2.6.3-4) by $\left|v\left(p_{i}\right)\right\rangle$.

We have $\omega(p)-\omega=0$ for $p=p_{j}$. Therefore, for each $j \in\{1,2, \ldots, m\}$ and each $l$, we get a consistency condition from (2.6.4). We have checked extensively that these consistency conditions are indeed satisfied. An analytic proof is outside the limited scope of this paper.

\section{The highest weight vector and descendants in $W$}

\subsection{Formal $U_{q}(\widehat{\mathfrak{s l}}(2))$ action}

To recall, the object of our interest is the semi infinite tensor product $W$ (1.2.2), of copies of the 2-dimensional irreducible representation $V$, of $U_{q}(\widehat{\mathfrak{s l}}(2))$. We can think of the weight vectors $(+)$ and $(-)$ of $V$ as spin-up and spin-down, or equivalently, as spin- $\frac{1}{2}$ and spin- $\frac{-1}{2}$.

The action of the generators of $U_{q}(\widehat{\mathfrak{s l}}(2))$ on $V$ is as follows:

$$
\begin{aligned}
& f_{0}(+)=e_{1}(+)=0, f_{0}(-)=e_{1}(-)=(+), \\
& f_{1}(+)=e_{0}(+)=(-), f_{1}(-)=e_{0}(-)=0, \\
& t_{1}(+)=t_{0}^{-1}(+)=q(+), t_{1}(-)=t_{0}^{-1}(-)=q^{-1}(-) .
\end{aligned}
$$

Notice that the generators form pairs that have the same action on the spin variables.

In our conventions, co-multiplication is of the form:

$$
\begin{aligned}
& \Delta\left(e_{i}\right)=e_{i} \otimes 1+t_{i}^{-1} \otimes e_{i}, \\
& \Delta\left(f_{i}\right)=f_{i} \otimes t_{i}+1 \otimes f_{i}, \\
& \Delta\left(t_{i}\right)=t_{i} \otimes t_{i} .
\end{aligned}
$$

Formally, the action of $e_{i}, f_{i}$ and $t_{i}$ on $W$ is as follows:

$$
\begin{aligned}
& \Delta^{(\infty)}\left(e_{i}\right)=e_{i} \otimes 1 \otimes 1 \otimes \cdots+t_{i}^{-1} \otimes e_{i} \otimes 1 \otimes \cdots+\cdots, \\
& \Delta^{(\infty)}\left(f_{i}\right)=f_{i} \otimes t_{i} \otimes t_{i} \otimes \cdots+1 \otimes f_{i} \otimes t_{i} \otimes \cdots+\cdots, \\
& \Delta^{(\infty)}\left(t_{i}\right)=t_{i} \otimes t_{i} \otimes t_{i} \otimes \cdots .
\end{aligned}
$$


From now on we abbreviate $\Delta^{(\infty)}\left(e_{i}\right), \Delta^{(\infty)}\left(f_{i}\right)$ and $\Delta^{(\infty)}\left(t_{i}\right)$ to $e_{i}, f_{i}$ and $t_{i}$, respectively.

We have $t_{0} t_{1}=1$ on $V$. Since we are aiming at constructing the level-1 vacuum representation in $W$, we define

$$
t_{0}|p\rangle=q^{1-2 s(p)}|p\rangle, \quad t_{1}|p\rangle=q^{2 s(p)}|p\rangle .
$$

Therefore, $t_{0} t_{1}=q$ on $W$.

Consider a formal element of $W$ :

$$
|v\rangle=\sum_{p} c(p, v)|p\rangle
$$

Our point is that, at $q \neq 0$, the physical states are infinite linear superpositions of paths in $W$. We use the convension $c(0, v)=0$.

Let us introduce a partial net spin $s_{k}(p)$ by

$$
s_{k}(p)=\sum_{1 \leq i \leq k-1} \frac{1}{2} p(i) .
$$

We define a map $\sigma_{k}^{ \pm}: \mathcal{P} \rightarrow \mathcal{P} \sqcup\{0\}$ by

$$
\begin{aligned}
\sigma_{k}^{ \pm}(p) & =0 & & \text { if } p(k)= \pm \\
\sigma_{k}^{ \pm}(p)(j) & =p(j) & & \text { if } j \neq k \text { and } p(k)=\mp \\
& =( \pm) & & \text { if } j=k \text { and } p(k)=\mp .
\end{aligned}
$$

Note that $s\left(\sigma_{k}^{ \pm}(p)\right)=s(p) \pm 1$.

Formally, the actions of $e_{i}$ and $f_{i}$ given by (3.1.1) can be expressed as follows:

$$
\begin{aligned}
& c\left(p, e_{0} v\right)=\sum_{k} q^{2 s_{k}(p)} c\left(\sigma_{k}^{+}(p), v\right), \\
& c\left(p, e_{1} v\right)=\sum_{k} q^{-2 s_{k}(p)} c\left(\sigma_{k}^{-}(p), v\right), \\
& c\left(p, f_{0} v\right)=\sum_{k} q^{2-2 s(p)+2 s_{k}(p)} c\left(\sigma_{k}^{-}(p), v\right), \\
& c\left(p, f_{1} v\right)=\sum_{k} q^{1+2 s(p)-2 s_{k}(p)} c\left(\sigma_{k}^{+}(p), v\right) .
\end{aligned}
$$

These sums contain infinitely many terms. Therefore, if we take an arbitrary element of the form (3.1.2), the right-hand-side of (3.1.3) is not always well-defined. We overcome this difficulty as follows:

Firstly, we make use of the fact that the generators of $U_{q}(\widehat{\mathfrak{s l}}(2))$ come in pairs, $\left(e_{0}, f_{1}\right)$ and $\left(e_{1}, f_{0}\right)$, that generate identical paths in the sum (3.1.3). The only difference is in the factors of $q$ that they obtain. But we will see that these factors of $q$ are independent of $k$ if $k$ is large. Hence, though the action of a single generator 
gives rise to an infinite number of terms, the actions of certain linear combinations of two generators, which we will denote by $g_{+}$and $g_{-}$in the below, generate only a finite number of terms in the sum.

Secondly, if we restrict our attention to the highest weight vector and the descendants, we can compute the actions of the generators in closed form by using the highest weight condition (3.2.2) and the usual commutation relations among the Chevalley generators.

Proposition 3.1 Define

$$
g_{+}=f_{0}-t_{1} e_{1}, g_{-}=f_{1}-t_{0} e_{0} .
$$

The action of these operators on $W$ is given by the following finite sum.

$$
\begin{aligned}
& c\left(p, g_{+} v\right)=\left(1-q^{2}\right) \sum_{k}\left[-1+2 s(p)-2 s_{k}(p)\right] c\left(\sigma_{k}^{-}(p), v\right), \\
& c\left(p, g_{-} v\right)=\left(1-q^{2}\right) \sum_{k}\left[-2 s(p)+2 s_{k}(p)\right] c\left(\sigma_{k}^{+}(p), v\right),
\end{aligned}
$$

where $[n]=\frac{q^{n}-q^{-n}}{q-q^{-1}}$.

This is immediate from (3.1.3). The finiteness follows from the fact that the asymptotic values of $s_{k}(p)$ in (3.1.4) and (3.1.5) when $k \rightarrow \infty$ are $s(p)-\frac{1}{2}$ and $s(p)$.

\subsection{The highest weight vector}

By the highest weight vector we mean a linear combination of paths

$$
|\mathrm{vac}\rangle=\sum_{k} c(p, \mathrm{vac})|p\rangle
$$

that satisfies

$$
e_{0}|\mathrm{vac}\rangle=0, e_{1}|\mathrm{vac}\rangle=0 \text {. }
$$

Since we are aiming at the embedding

$$
V\left(\Lambda_{0}\right) \rightarrow W,
$$

we also require

$$
t_{0}|\mathrm{vac}\rangle=q|\mathrm{vac}\rangle, t_{1}|\mathrm{vac}\rangle=|\mathrm{vac}\rangle, f_{0}^{2}|\mathrm{vac}\rangle=0, f_{1}|\mathrm{vac}\rangle=0 .
$$

We have, in particular, that $c(p$, vac $)=0$ unless $s(p)=0$. For simplicity we abbreviate $c(p, \operatorname{vac})$ to $c(p)$ in this section. In order to get $c(p)$, we may solve (3.2.2) perturbatively in $q$, just as we did for (2.5.1). However, using the observation we made above, it is possible to compute $c(p)$ exactly. 
Example. $\quad$ Let us compute $c(1)$. We use $\left(f_{1}-t_{0} e_{0}\right) \mid$ vac $\rangle=0$. Applying (3.1.5) for $p=(---+-+\cdots)$, we get

$$
[2] c(1)+c(\varnothing)=0 .
$$

Hence $c(1)=-\varepsilon$.

In general, we proceed as follows. The equations (3.2.2-3) imply

$$
g_{+}^{2}|\mathrm{vac}\rangle=0, g_{-}|\mathrm{vac}\rangle=0 .
$$

Therefore, by using (3.1.4-5) we have

$$
\begin{aligned}
& \sum_{k}\left[2+2 s_{k}(p)\right] c\left(\sigma_{k}^{+}(p)\right)=0 \text { for } p \text { such that } s(p)=-1 \\
& \sum_{k_{1}<k_{2}}\left[2-2 s_{k_{1}}(p)\right]\left[3-2 s_{k_{2}}(p)\right] c\left(\sigma_{k_{1}}^{-} \sigma_{k_{2}}^{-}(p)\right)=0 \text { for } p \text { such that } s(p)=2 .
\end{aligned}
$$

The question now is whether we can solve the above equations simultaneously. In the following, we show that the system of linear equations (3.2.5-6) is of blocktriangular form. We conjecture that the diagonal blocks are invertible. Let $\mathcal{P}_{l, s}$ be the set of paths $p$ such that $l(p)=l$ and $s(p)=s$. Then we have bijections

$$
\begin{aligned}
\mathcal{P}_{l, 0} & \sim \mathcal{P}_{l,-1} \text { if } l \geq 2 \text { is even, } \\
& \sim \mathcal{P}_{l, 2} \quad \text { if } l \geq 3 \text { is odd. }
\end{aligned}
$$

In fact, the cardinality is equal to $\left(\begin{array}{c}l-1 \\ {\left[\frac{l}{2}\right]-1}\end{array}\right)$. The bijection from $\mathcal{P}_{l,-1}(l$ : even $)$ or $\mathcal{P}_{l, 2}(l:$ odd $)$ to $\mathcal{P}_{l, 0}$ is constructed as follows. Take $p \in \mathcal{P}_{l,-1}$ or $p \in \mathcal{P}_{l, 2}$. Consider $(-p(1),-p(2), \ldots,-p(l-1))$, and apply the procedure (2.4.1) to this finite sequence, then we get a list $a \in \mathcal{P}_{l, 0}$. For example, the correspondence up to $l=4$ goes as

$$
\begin{aligned}
& (---+-+\cdots) \leftrightarrow(1), \\
& (++++-+\cdots) \leftrightarrow(01), \\
& (+----+\cdots) \leftrightarrow(001), \\
& (-+---+\cdots) \leftrightarrow(11), \\
& (--+--+\cdots) \leftrightarrow(12) .
\end{aligned}
$$

If $l\left(\sigma_{k}^{+}(p)\right)>l(p)$ in $(3.2 .5)$, then we have $s_{k}(p)=-1$, and if $l\left(\sigma_{k_{1}}^{-} \sigma_{k_{2}}^{-}(p)\right)>l(p)$ in (3.2.6), then we have $s_{k_{2}}(p)=\frac{3}{2}$. Therefore, the system of linear equations is of block-triangular form with respect to the length of paths. 
The coefficients of the first few equations are as follows.

$$
\begin{aligned}
& \begin{array}{llllllllll}
\varnothing & 1 & 01 & 001 & 11 & 12 & 101 & 0001 & 011 & 012
\end{array} \\
& \begin{array}{l|cccccccccc}
-- & 1 & {[2]} & 0 & 0 & 0 & 0 & 0 & 0 & 0 & 0 \\
+++ & {[2]} & 1 & {[2]^{2}} & 0 & 0 & 0 & 0 & 0 & 0 & 0 \\
+--- & 0 & 1 & 0 & 0 & {[2]} & {[3]} & 0 & 0 & 0 & 0 \\
-+-- & 1 & 0 & 0 & {[2]} & 0 & {[2]} & 0 & 0 & 0 & 0
\end{array} \\
& \begin{array}{l|llllllllll}
--+- & 0 & 0 & 1 & 1 & {[2]} & 0 & 0 & 0 & 0 & 0
\end{array}
\end{aligned}
$$

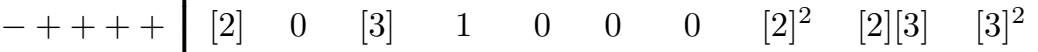

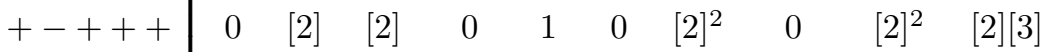

$$
\begin{aligned}
& ++-++\left(\begin{array}{llllllllll}
{[2]} & 1 & 0 & 0 & 0 & 1 & {[2]} & {[2]^{2}} & 0 & {[2]^{2}}
\end{array}\right.
\end{aligned}
$$

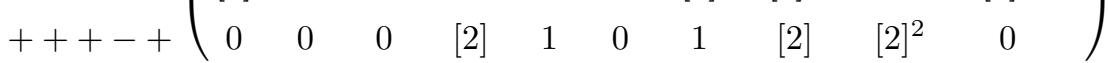

The following is the list of $c(p)$ for $|\mathrm{vac}\rangle$ up to $l(p)=6$.

$l=0$

$|\varnothing\rangle \quad 1$

$l=2$

$|1\rangle$

$-\varepsilon$

$l=3$

$|01\rangle$

$\varepsilon^{3}-\varepsilon$

$l=4$

$|001\rangle \quad-\varepsilon^{5}+\varepsilon^{3}-\varepsilon$

|11) $\quad \varepsilon^{6}-2 \varepsilon^{4}+2 \varepsilon^{2}$

$|12\rangle \quad \varepsilon^{5}-\varepsilon^{3}$

$l=5$

|0001) $\quad \varepsilon^{9}-\varepsilon^{7}+\varepsilon^{3}-\varepsilon$

|011) $\quad \varepsilon^{10}-3 \varepsilon^{8}+5 \varepsilon^{6}-5 \varepsilon^{4}+2 \varepsilon^{2}$

$|012\rangle \quad \varepsilon^{9}-3 \varepsilon^{7}+3 \varepsilon^{5}-\varepsilon^{3}$

$|101\rangle \quad-2 \varepsilon^{8}+3 \varepsilon^{6}-2 \varepsilon^{4}+\varepsilon^{2}$

$l=6$

|00001) $\quad \varepsilon^{13}-5 \varepsilon^{11}+7 \varepsilon^{9}-4 \varepsilon^{7}+\varepsilon^{3}-\varepsilon$

|0011) $\quad \varepsilon^{14}-2 \varepsilon^{12}+3 \varepsilon^{10}-6 \varepsilon^{8}+8 \varepsilon^{6}-5 \varepsilon^{4}+2 \varepsilon^{2}$

|0012 $\quad \varepsilon^{13}-2 \varepsilon^{11}+4 \varepsilon^{9}-5 \varepsilon^{7}+3 \varepsilon^{5}-\varepsilon^{3}$

$|0101\rangle-2 \varepsilon^{12}+6 \varepsilon^{10}-8 \varepsilon^{8}+6 \varepsilon^{6}-3 \varepsilon^{4}+\varepsilon^{2}$

$|1001\rangle-\varepsilon^{14}+5 \varepsilon^{12}-8 \varepsilon^{10}+7 \varepsilon^{8}-3 \varepsilon^{6}+\varepsilon^{2}$

$|111\rangle-\varepsilon^{15}+4 \varepsilon^{13}-10 \varepsilon^{11}+17 \varepsilon^{9}-19 \varepsilon^{7}+13 \varepsilon^{5}-5 \varepsilon^{3}$

$|112\rangle-\varepsilon^{14}+4 \varepsilon^{12}-10 \varepsilon^{10}+13 \varepsilon^{8}-9 \varepsilon^{6}+3 \varepsilon^{4}$

$|121\rangle-2 \varepsilon^{14}+7 \varepsilon^{12}-11 \varepsilon^{10}+11 \varepsilon^{8}-8 \varepsilon^{6}+3 \varepsilon^{4}$

$|122\rangle-2 \varepsilon^{13}+7 \varepsilon^{11}-11 \varepsilon^{9}+9 \varepsilon^{7}-3 \varepsilon^{5}$

|123〉 $-\varepsilon^{12}+3 \varepsilon^{10}-3 \varepsilon^{8}+\varepsilon^{6}$

\subsection{Descendants}


The descendants are by definition the vectors obtained from the highest weight vector $\mid$ vac $\rangle$ by the actions of $f_{0}$ and $f_{1}$. We give a recursive formula for getting the descendants. Note first that by (3.1.4-5) the action of $g_{+}$and $g_{-}$are finite on the space of vectors of the form (3.1.2). In particular, the space of vectors created upon $|\mathrm{vac}\rangle$, which we denote by $U$, is well-defined. Let us give a recursive formula for the actions on $U$, of the Chevalley generators of $U_{q}(\widehat{\mathfrak{s l}}(2))$. We use the notation $\{x\}=\frac{x-x^{-1}}{q-q^{-1}}$. Let $|v\rangle \in U$ be a monomial of the form

$$
|v\rangle=g_{m_{1}} \cdots g_{m_{k}}|\mathrm{vac}\rangle .
$$

If $k \neq 0$, we set $\left|v^{\prime}\right\rangle=g_{m_{2}} \cdots g_{m_{k}} \mid$ vac $\rangle$. We have

$$
\begin{array}{rlrl}
t_{0}|v\rangle & =q^{1-2 \sum_{i} m_{i}}|v\rangle, & \\
t_{1}|v\rangle & =q^{2 \sum_{i} m_{i}}|v\rangle, & & \\
e_{0}|v\rangle & =0 & & \text { if } k=0, \\
& =\left(\left\{t_{0}\right\}+f_{0} e_{0}-e_{0} t_{1} e_{1}\right)\left|v^{\prime}\right\rangle & & \text { if } m_{1}=+, \\
& =\left(f_{1} e_{0}-e_{0} t_{0} e_{0}\right)\left|v^{\prime}\right\rangle & & \text { if } m_{1}=-, \\
e_{1}|v\rangle & =0 & & \text { if } k=0, \\
& =\left(f_{0} e_{1}-e_{1} t_{1} e_{1}\right)\left|v^{\prime}\right\rangle & & \text { if } m_{1}=+, \\
& =\left(\left\{t_{1}\right\}+f_{1} e_{1}-e_{1} t_{0} e_{0}\right)\left|v^{\prime}\right\rangle & & \text { if } m_{1}=-, \\
f_{0}|v\rangle & =\left(g_{+}+t_{1} e_{1}\right)|v\rangle, & & \\
f_{1}|v\rangle & =\left(g_{-}+t_{0} e_{0}\right)|v\rangle . & &
\end{array}
$$

Let $d$ be the derivation of $U_{q}(\widehat{\mathfrak{s l}}(2))$ that satisfies

$$
\left[d, t_{i}\right]=0,\left[d, e_{i}\right]=-\delta_{i 0} e_{i},\left[d, f_{i}\right]=\delta_{i 0} f_{i} .
$$

We define the action of $d$ on $U$ by $d \mid$ vac $\rangle=0$.

Take $s \in \mathbf{Z}$ and $\omega \in \mathbf{Z}_{>0}$. An descendant of spin $s$ and energy $\omega$ is a vector $|v\rangle$ of $U$ satisfying $t_{1}|v\rangle=q^{2 s}|v\rangle, d|v\rangle=\omega|v\rangle$. Let us denote the space of such vectors by $U_{s \omega}$.

Example. Let us calculate a few coefficients of $f_{0} \mid$ vac $\rangle$ and $f_{1} f_{0} \mid$ vac $\rangle$. We have

$$
f_{0}|\mathrm{vac}\rangle=g_{+}|\mathrm{vac}\rangle .
$$

From (3.1.4) a first few coefficients read as

$$
\begin{aligned}
c\left(++-+-+-+\cdots, f_{0}|\operatorname{vac}\rangle\right) & =\left(1-q^{2}\right) c(\varnothing) \\
& =\left(1-q^{2}\right) \cdot 1 \\
c\left(+-++-+-+\cdots, f_{0}|\operatorname{vac}\rangle\right) & =\left(1-q^{2}\right)\{c(01)+c(1)\} \\
& =\left(1-q^{2}\right)\left(-2 \varepsilon+\varepsilon^{3}\right), \\
c\left(+-+-++-+\cdots, f_{0}|\operatorname{vac}\rangle\right) & =\left(1-q^{2}\right)\{c(011)+c(101)+c(11)\} \\
& =\left(1-q^{2}\right)\left(5 \varepsilon^{2}-9 \varepsilon^{4}+9 \varepsilon^{6}-5 \varepsilon^{8}+\varepsilon^{10}\right), \\
c\left(-+++-+-+\cdots, f_{0}|\operatorname{vac}\rangle\right) & =\left(1-q^{2}\right)\{[2] c(01)+c(\varnothing)\} \\
& =\left(1-q^{2}\right) \varepsilon^{2} .
\end{aligned}
$$


Similarly, we have

$$
f_{1} f_{0}|\mathrm{vac}\rangle=g_{-} g_{+}|\mathrm{vac}\rangle+q|\mathrm{vac}\rangle .
$$

A first few coefficients are

$$
\begin{aligned}
& c\left(\varnothing, f_{1} f_{0}|\mathrm{vac}\rangle\right)=q, \\
& c\left(1, f_{1} f_{0}|\mathrm{vac}\rangle\right)=\left(1-q^{2}\right)^{2}-q \varepsilon, \\
& c\left(01, f_{1} f_{0}|\mathrm{vac}\rangle\right)=-\left(1-q^{2}\right)^{2} \varepsilon^{2}-q\left(\varepsilon-\varepsilon^{3}\right), \\
& c\left(12, f_{1} f_{0}|\mathrm{vac}\rangle\right)=\left\{2\left(1-q^{2}\right)^{2}-q \varepsilon\right\}\left(\varepsilon^{2}-\varepsilon^{4}\right) .
\end{aligned}
$$

4. Identity between the eigenvectors of $\mathcal{H}$ and the $U_{q}(\widehat{\mathfrak{s l}}(2))$-weight vectors

\subsection{Conjecture}

In $\S 2$ we developed a scheme for diagonalizing the CTM Hamiltonian $\mathcal{H}_{r e}$ acting on $W$, and introduced the space of eigenvectors $H_{s \omega}$ of spin $s$ and energy $\omega$. In $\S 3$ we developed a scheme for realizing the level-1 vacuum representation of $U_{q}(\widehat{\mathfrak{s l}}(2))$ in $W$, and introduced the space of weight vectors $U_{s \omega}$ of spin $s$ and energy $\omega$.

We set forth

Conjecture 4.1 The eigenvectors of $\mathcal{H}_{r e}$ form the level-1 vacuum representation $V\left(\Lambda_{0}\right)$ of the quantum affine Lie algebra $U_{q}(\widehat{\mathfrak{s l}}(2))$, i.e., we have $H_{s \omega}=U_{s \omega}$.

As a corollary we deduce that

$$
\mathcal{H}_{\text {re }}=d
$$

on $U$. ( $d$ is the derivation (3.3.2).)

In this section we report on our checks on this conjecture. We used mostly the following data.

Data for $H_{s \omega}$.

Data 1: The coefficients $c_{l}(p, \phi)$, for $p=(\underbrace{0 \cdots 0}_{k-1} 1)$ and $0 \leq l \leq 5,1 \leq k \leq$ $41-l(l+1)$, obtained by solving (2.5.3).

Data 2: The coefficients $c_{l}\left(p_{1}, v\left(p_{2}\right)\right)$, for $p_{1}, p_{2} \in\{p \mid s(p)=0, \omega(p) \leq 4\}$ and $0 \leq l \leq 2$, obtained by solving (2.6.4). (There are 12 such paths.)

Data for $U_{s \omega}$.

Data 3: The coefficients $c(p$, vac $)$, for $p$ such that $l(p) \leq 9$, obtained by solving (3.2.4-5). (There are 126 such paths.)

Data 4: The coefficients $c\left(p_{1}, v\left(p_{2}\right)\right)$ for $p_{1}, p_{2} \in\{p \mid s(p)=0, \omega(p) \leq 3\}$, obtained by the action (3.3.1). (There are seven such paths.) 


\subsection{Checks}

We made the following five different checks.

Check 1: Comparison of Data 1 and Data 3.

This check is particularly important because $c(\underbrace{0 \cdots 0}_{k-1} 1, \phi)$ are the renormalization terms $R_{k}$ in (2.5.4). Note that Data 1 contain only first 6 nontrivial coefficients $(0 \leq k \leq 5)$, but of longer paths than Data 2, which are exact but limited to the paths of length less than or equal to 9 . We checked the coincidence of these two sets of data within the common region of accuracy and length.

Here are the relevant part of Data 3 in addition to those given in $\S 3$.

$$
\begin{array}{ll}
|000001\rangle & 2 \epsilon^{19}-9 \epsilon^{17}+19 \epsilon^{15}-21 \epsilon^{13}+12 \epsilon^{11}-2 \epsilon^{9}-\epsilon^{7}+\epsilon^{3}-\epsilon \\
|0000001\rangle & -2 \epsilon^{25}+17 \epsilon^{23}-64 \epsilon^{21}+146 \epsilon^{19}-228 \epsilon^{17}+247 \epsilon^{15} \\
& -179 \epsilon^{13}+79 \epsilon^{11}-16 \epsilon^{9}-\epsilon^{7}+\epsilon^{3}-\epsilon \\
|00000001\rangle & 6 \epsilon^{33}-47 \epsilon^{31}+188 \epsilon^{29}-499 \epsilon^{27}+968 \epsilon^{25}-1433 \epsilon^{23}+1638 \epsilon^{21} \\
& -1420 \epsilon^{19}+893 \epsilon^{17}-375 \epsilon^{15}+86 \epsilon^{13}-2 \epsilon^{11}-2 \epsilon^{9}-\epsilon^{7}+\epsilon^{3}-\epsilon
\end{array}
$$

Data 1 and Data 3 also show a strong evidence that $c_{l}(\underbrace{0 \cdots 0}_{k} 1, \phi)$ is independent of $k$ for $k \geq \max (2 l-1,0)$. In general, we conjecture that the following two asymptotic properties. We use the list notation (2.4.1) for the spin-0 paths.

(A) For any path $p$, the coefficient $c_{l}(\underbrace{0 \cdots 0}_{k} p, \phi)$ is independent of $k$ for $k \geq \max (2 l-$ $1,0)$.

(B) For any paths $p$ and $p^{\prime}$, we have $c_{l}(\underbrace{0 \cdots 0}_{k} p^{\prime}, \phi)=\sum_{j} c_{j}(p, \phi) c_{l-j}(\underbrace{0 \cdots 0}_{k+l(p)} p^{\prime}, \phi)$ for $k \geq 2 l+1$. The case when $p^{\prime}=(1)$ was already mentioned in $\S 2$.

Check 2: $\mathcal{H}|\mathrm{vac}\rangle=0$ by using Data 3 .

Let us abbreviate $c(p, \operatorname{vac})$ by $c(p)$. Given a spin-0 path, we wish to check

$$
\left\{\omega(p)-\varepsilon \sum_{k \geq 1} k c(\underbrace{0 \cdots 0}_{k-1} 1)\right\} c(p)+\varepsilon \sum_{k \geq 1} k c\left(Q_{k} p\right)=0 .
$$

Let us discuss a simple example.

Example. Let us consider (4.2.1) for $p=(+--+-+\cdots)$. We have $\omega(1)=1$, and $c(\underbrace{0 \cdots 0}_{k-1} 1)=-\varepsilon,-\varepsilon+\varepsilon^{3},-\varepsilon+\varepsilon^{3}-\varepsilon^{5}, \ldots$ for $k=1,2,3, \ldots$, as given previously. The second sum in $(4.2 .1)$ reads as $c(\varnothing)+3 c(11)+4 c(101)+5 c(1001)+\cdots$. The relevant terms which are not included in the table in $\S 3$ are

$$
\begin{aligned}
& -3 \epsilon^{18}+14 \epsilon^{16}-34 \epsilon^{14}+48 \epsilon^{12}-40 \epsilon^{10}+18 \epsilon^{8}-3 \epsilon^{6}-\epsilon^{4}+\epsilon^{2} \\
& 2 \epsilon^{26}-17 \epsilon^{24}+64 \epsilon^{22}-148 \epsilon^{20}+240 \epsilon^{18}-280 \epsilon^{16} \\
& +234 \epsilon^{14}-139 \epsilon^{12}+58 \epsilon^{10}-16 \epsilon^{8}+3 \epsilon^{6}-\epsilon^{4}+\epsilon^{2}
\end{aligned}
$$


$|1000001\rangle$

$$
\begin{aligned}
& -8 \epsilon^{32}+60 \epsilon^{30}-242 \epsilon^{28}+682 \epsilon^{26}-1450 \epsilon^{24}+2387 \epsilon^{22}-3073 \epsilon^{20} \\
& +3082 \epsilon^{18}-2365 \epsilon^{16}+1341 \epsilon^{14}-528 \epsilon^{12}+127 \epsilon^{10}-13 \epsilon^{8}-\epsilon^{4}+\epsilon^{2}
\end{aligned}
$$

Since we can take account of only finitely many terms, we must estimate the accuracy we can expect. We use the paths of length less than or equal to 9. Admitting the asymptotic factorization (B) mentioned above, we can expect the cancellation $\bmod \varepsilon^{9}$. This is in fact true.

Following a similar procedure we have checked that $\mathcal{H}_{\text {re }} \mid$ vac $\rangle=0$ within the accuracy expected from Data 3, and also that the state $f_{0} \mid$ vac $\rangle$ is the first excited state of $\mathcal{H}_{r e}$ with eigenvalue 1 . However, because the coefficients are much more complicated for this state, we could perform the computations only for all paths up to length 8 , and to lowest non-trivial order in $\varepsilon$.

Check 3: Comparison of Data 2 and Data 4.

The consistency of the equation (2.6.4) is not obvious, especially when the eigenvalue has non-trivial multiplicity. Therefore, we computed Data 2 rather extensively, i.e., the energy up to 4 , as a check of (2.6.4) itself. The result was in favor of our consistency assumption.

Then we checked the coincidence of Data 2 and Data 4 within the common region of accuracy and energy. We omit the details of this check, since the most of the data were created only in the memory of our computer.

Check 4: $e_{0}|\mathrm{vac}\rangle=e_{1}|\mathrm{vac}\rangle=0$ by using Data 3 .

Recall that the reason why we could evaluate coefficients $c(p$, vac $)$ exactly, in $\S 3$, was that we made use of (3.2.4) instead of (3.2.2-3). In Check 4 that follows, we have to act with single generators $e_{0}$ and $e_{1}$, so we end up with infinite series (3.1.3), and must turn to perturbation theory.

The equations read as

$$
\begin{aligned}
& \sum_{k} q^{2 s_{k}(p)} c\left(\sigma_{k}^{+}(p), v\right)=0 \text { for } s(p)=-1 \\
& \sum_{k} q^{-2 s_{k}(p)} c\left(\sigma_{k}^{-}(p), v\right)=0 \text { for } s(p)=1
\end{aligned}
$$

Let us discuss a simple example.

Example. $\quad$ Let us cosider (4.2.3) for $p=(++-+-+\cdots)$. We are to check

$$
c(1)+c(12)+c(122)+\cdots=-q .
$$

The datum available in addition to those given in $\S 3$ is

$$
6 \epsilon^{25}-39 \epsilon^{23}+128 \epsilon^{21}-277 \epsilon^{19}+429 \epsilon^{17}-485 \epsilon^{15}+396 \epsilon^{13}-225 \epsilon^{11}+81 \epsilon^{9}-14 \epsilon^{7}
$$

Assuming that

$$
c(1 \underbrace{2 \cdots 2}_{k})=0 \bmod \varepsilon^{2 k+1},
$$


we can expect

$$
c(1)+c(12)+\cdots+c(1222)=-\varepsilon-\varepsilon^{3}-2 \varepsilon^{5}-5 \varepsilon^{7} \bmod \varepsilon^{9},
$$

which is certainly true.

In general, we have checked (4.2.2-3) up to the accuracy that are expected from the data for the paths of length less than or equal to 9 .

Check 5: (4.2.4) by using some additional data.

Since we explicitly know the coefficients of only a finite number of paths: those up to length 9 , the accuracy of the cancellations is rather limited. To make our check sure we computed a few more terms in (4.2.4) by solving (2.5.3):

$$
\begin{aligned}
c(1222) & =-84 \varepsilon^{9}+770 \varepsilon^{11}-3529 \varepsilon^{13}+\cdots, \\
c(12222) & =-594 \varepsilon^{11}+7722 \varepsilon^{13}+\cdots, \\
c(1222222) & =-4719 \varepsilon^{13}+\cdots
\end{aligned}
$$

By using this we checked (4.2.4) $\bmod \varepsilon^{15}$.

\subsection{The commutation relations}

Since we are interested in the relation between $\mathcal{H}_{r e}$, and $U_{q}(\widehat{\mathfrak{s l}}(2))$, we wish to evaluate their commutators. We have

$$
\left[\mathcal{H}_{r e}, e_{i}\right]=-\delta_{i 0} e_{i},\left[\mathcal{H}_{r e}, f_{i}\right]=\delta_{i 0} f_{i} .
$$

Each commutator maps a given path to an infinite superposition of paths. We will show that in each case of (4.3.1), the commutator in the left-hand-side gives the same superposition and the Chevalley generator in the right-hand-side, for each choice of a path.

We neglect all scalar terms in $\mathcal{H}_{r e}$, since their contributions trivially cancel out in the commutators. We also discard the term proportional to $S$, since its commutators with $e_{i}, f_{i}$ are known. So, we set $\mathcal{H}^{\prime}=P^{\prime}+\varepsilon Q$, and compute its commutators with $e_{i}, f_{i}$.

From $\S 2$, we recall that $P^{\prime}=\sum_{k=1}^{\infty} k P_{k}^{\prime}$, and $Q=\sum_{k=1}^{\infty} k Q_{k}$, where $P_{k}^{\prime}$ and $Q_{k}$ are bi-local operators: they act on pairs of neighbouring spins (2.2.1-2). From $\S 3$, we recall the action of the co-multiplication of $e_{i}$ and $f_{i}$ on a path (3.1.1). The latter is the sum of an infinite number of semi-infinite tensor products. Let us use $e_{i k}, f_{i k}$ to denote single semi-infinite tensor products, with a generator in the $k$-th position, e.g.,

$$
e_{1 k}=\underbrace{t_{i}^{-1} \otimes \ldots \otimes t_{i}^{-1}}_{k-1} \otimes e_{1} \otimes 1 \otimes \ldots
$$

We will derive $\left[\mathcal{H}_{r e}, e_{1}\right]$ in some detail as an example. Consider $\left[\mathcal{H}_{k}^{\prime}, e_{1 k^{\prime}}\right]$ where $\mathcal{H}_{k}^{\prime}=P_{k}^{\prime}+\varepsilon Q_{k}$. It vanishes unless $k^{\prime}=k, k+1$. Therefore, we have two cases to 
consider: $k^{\prime}=k$ and $k^{\prime}=k+1$. We start with $k^{\prime}=k$. We compute the action of the commutator on the 4 possible spin-configurations $\left(s s^{\prime}\right)\left(s, s^{\prime}= \pm\right)$ on the $k$-th and the $(k+1)$-the components. We start with $(--)$ :

$$
\begin{aligned}
\left(P_{k}^{\prime}+\varepsilon Q_{k}\right) e_{1 k}(--) & =\left(P_{k}^{\prime}+\varepsilon Q_{k}\right) q^{-2 s_{k}(p)}(+-) \\
& =q^{-2 s_{k}(p)}(+-)+\varepsilon q^{-2 s_{k}(p)}(-+), \\
-e_{1 k}\left(P_{k}^{\prime}+\varepsilon Q_{k}\right)(--) & =-q^{-2 s_{k}(p)}(+-) .
\end{aligned}
$$

Therefore we have, $\left[\mathcal{H}_{k}^{\prime}, e_{1 k}\right](--)=\varepsilon q^{-2 s_{k}(p)}(-+)$.

Similarly, we obtain the following as a whole:

$$
\begin{aligned}
& {\left[\mathcal{H}_{k}^{\prime}, e_{1 k}\right](--)=\varepsilon q^{-2 s_{k}(p)}(-+),} \\
& {\left[\mathcal{H}_{k}^{\prime}, e_{1 k}\right](-+)=q^{-2 s_{k}(p)}(++),} \\
& {\left[\mathcal{H}_{k}^{\prime}, e_{1 k}\right](+-)=-\varepsilon q^{-2 s_{k}(p)}(++),} \\
& {\left[\mathcal{H}_{k}^{\prime}, e_{1 k}\right](++)=0 .}
\end{aligned}
$$

Repeating the same excercise for $e_{1 k+1}$, we obtain:

$$
\begin{aligned}
& {\left[\mathcal{H}_{k}^{\prime}, e_{1 k+1}\right](--)=\varepsilon q^{-2 s_{k}+1}(+-)-q^{-2 s_{k}+1}(-+),} \\
& {\left[\mathcal{H}_{k}^{\prime}, e_{1 k+1}\right](-+)=-\varepsilon q^{-2 s_{k}-1}(++),} \\
& {\left[\mathcal{H}_{k}^{\prime}, e_{1 k+1}\right](+-)=0} \\
& {\left[\mathcal{H}_{k}^{\prime}, e_{1 k+1}\right](++)=0}
\end{aligned}
$$

Note that in (4.3.2-9) the bracket changes a $(-)$ into a $(+)$ (if the result is not just zero.)

Next, we will consider the action of the commutator $\left[\mathcal{H}_{k}^{\prime}, e_{1 k^{\prime}}\right]$ on an arbitrary but fixed path, say $p$, and collect the contributions given by (4.3.2-9) such that the change of the $(-)$ into the $(+)$ takes place at the $k$-th component.

Let us start with the case where the there spins at the $(k-1)$-th, the $k$-th and the $(k+1)$-th components are $(+-+)$. The contributions to $(+++)$ are from (4.3.3), (4.3.4) and (4.3.7), i.e., $-(k-1) \varepsilon q^{-2 s_{k-1}(p)}, k q^{-2 s_{k}(p)},-k \varepsilon q^{-2 s_{k}(p)-1}$, respectively. Summing up these three terms, and using $s_{k}(p)=s_{k-1}(p)+\frac{1}{2}$, we get $\varepsilon q^{-2 s_{k-1}(p)}$.

This is exactly the coefficient that we would obtain under the action of $\varepsilon q e_{1 k}$. This can be verified for the rest of the possible cases, and we deduce that

$$
\left[\mathcal{H}^{\prime}, e_{1}\right]=\varepsilon q e_{1} .
$$

We have to pause at this point to remark that the individual actions of the commutator give weights that contain the position $k$ as a factor. All such dependences neatly cancel out, once we sum the various contributions. When one performs the calculation, such cancellations are totally mysterious. 
But, they are precisely these cancellations that allow the final result to coincide with that obtained from the action of the Chevalley generator, and that contains no such multiplicative $k$-dependence.

The rest of the commutation relations can be proven in exactly the same way. We obtain:

$$
\begin{aligned}
{\left[\mathcal{H}^{\prime}, e_{0}\right] } & =-\varepsilon q^{-1} e_{0}, \\
{\left[\mathcal{H}^{\prime}, f_{0}\right] } & =\varepsilon q^{-1} f_{0}, \\
{\left[\mathcal{H}^{\prime}, f_{1}\right] } & =-\varepsilon q f_{1} .
\end{aligned}
$$

From these commutation relations, we can see that $\mathcal{H}_{r e}$ acts as a derivation of $U_{q}(\widehat{\mathfrak{s l}}(2))$.

As discussed above, these simple commutators is at this point unexpected and mysterious. Together with the direct checks that we discussed before, they encouraged us to present Conjecture 4.1.

Note added in proof. After finishing this work, [FT] was brought to our attention, where a study of CTM eigenvectors of the six-vertex model, both in the ferro-electric $\Delta>1$, and anti-ferro-electric $\Delta<-1$ regimes, is presented.

The connection between our eigenstates, and those obtained in $[\mathrm{FT}]$ remains to be understood. The point is that Frahm and Thacker are considering the CTM eigenstates on the whole line, while our CTM eigenstates are on the half line.

We also add [PS] in our reference, in which the commutation relations between the row-to-row transfer matrix Hamiltonian and $U_{q}(\mathfrak{s l}(2))$ is calculated on a finite lattice. Note that our commutation relations are between the CTM Hamiltonian and $U_{q}(\widehat{\mathfrak{s l}}(2))$.

\section{References}

[ABF $]$ G.E. Andrews, R.J. Baxter and P.J. Forrester, Eight-vertex SOS models and generalized Rogers-Ramanujan identities, J. Stat. Phys. 35 (1984), 193-266.

[B1] R.J. Baxter, Exactly Solved Models in Statistical Mechanics, 1982 Academic.

[B2] R.J. Baxter, Corner Transfer Matrices of the Eight-Vertex Model, J. Stat. Phys. 15 (1976), 485-503; 17 (1977), 1-14.

[Da1] B. Davies, Corner transfer matrices for the Ising model, Physica A 154 (1988), 1-20.

[Da2] B. Davies, On the spectrum of six-vertex corner transfer matrices, Physica A 159 (1989), 171-187.

[Dr] V.G. Drinfeld, Quantum groups, Proc. ICM Berkeley (1987), 798-820.

[DJKMO1] E. Date, M. Jimbo, A. Kuniba, T. Miwa and M. Okado, Exactly solvable SOS models: Local height probabilities and theta function identities, Nucl, 
Phys. Bbf 290 [FS20] (1987), 231-273, II. Proof of the star-triangle relation and combinatorial identities, Adv. Stud. Pure Math. 16 (1988), 17-122.

[DJKMO2] Date, E., Jimbo, M., Kuniba, A., Miwa, T. and Okado, M, One dimensional configuration sums in vertex models and affine Lie algebra characters, Lett. Math. Phys., 17 (1989), 69-77.

[DP] B. Davies and P. A. Pearce, Conformal invariance and critical spectrum of corner transfer matrices, J. Phys. A 23 (1990), 1295-1312.

[F] O. Foda, A relation between off-critical local height probabilities, and critical pertition functions in a finite geometry, preprint, Univ. of Nijmegen, 1991.

[IT] H. Itoyama and H.B. Thacker, Integrability and Virasoro symmetry of the noncritical Baxter/Ising model, preprint, Fermilab, 1988.

[J] M. Jimbo, Introduction to the Yang-Baxter equation, Inter. J. Mod. Phys. A, 4 (15) (1989), 3759-3777.

[JMMO] M. Jimbo, K.C. Misra, T. Miwa and M. Okado, Combinatorics of representations of $U_{q}(\hat{s} l(n))$ at $q=0$, Comm. Math. Phys. 136 (1991), 543-566.

[K] M. Kashiwara, Crystalizing the $q$-analogue of universal enveloping algebras, Commun. Math. Phys. 133 (1990), 249-260; On crystal bases of the qanalogue of universal enveloping algebras, Duke Math. J. 63 (1991), 465516 .

$\left[(\mathrm{KMN})^{2}\right]$ S.J. Kang, M. Kashiwara, K.C. Misra, T. Miwa, T. Nakashima and A. Nakayashiki, Affine crystals and vertex models, preprint, RIMS-828 (1991).

$[\mathrm{MM}]$ K.C. Misra and T. Miwa, Crystal base for the basic representation of $U_{q}(\hat{s} l(n))$, Commun. Math. Phys. 134 (1990), 79-88.

[SB] H. Saleur and M. Bauer, On some relations between local height probabilities and conformal invariance, Nucl. Phys. B 320 (1989), 591-624.

[SW] K. Sogo and M. Wadati, Boost operator and its application to quantum Gelfand-Levitan equation for Heisenberg-Ising chain with spin one-half, Prog. Theor. Phys. 69 (1983), 431.

[Te] M.G. Tetel'man, Lorentz group for two-dimensional integrable lattice systems, Zh. Eksp. Teor. Fiz. 82 (1982), 528-535.

[T] H.B. Thacker, Corner transfer matrices and Lorentz invariance on a lattice, Physica D 18 (1986), 348-359.

[TP] T.T. Truong and I. Peschel, Diagonalization of finite-size corner transfer matrices and related spin chains, Z. Phys. B 75 (1989), 119-125.

[FT] H. Frahm and H.B. Thacker, Corner transfer matrix eigenstates for the sixvertex model, preprint, University of Virginia, 1991.

[PS] V. Pasquier and H. Saleur, Common structures between finite systems and conformal field theories through quantum groups, Nucl. Phys. B 330 (1990), 523556 . 